\title{
Kolonkarzinom: Adjuvante Chemotherapie bei defekter Mismatch-Reparatur
}

\section{Patienten mit Kolonkarzinom im Stadium III mit defekter Mismatch- Reparatur profitieren nur von adjuvanter Chemotherapie, wenn sie Oxaliplatin enthält.}

$D^{i}$ ie adjuvante Chemotherapie mit 5-Fluorouracil (5-FU) reduziert das Rezidivrisiko von Patienten mit Kolonkarzinom im Stadium III. Bei StadiumII-Tumoren ist dieses Vorgehen jedoch umstritten. Zudem scheinen Tumoren mit einem Defekt in der Mismatch-Reparatur resistent gegenüber 5-FU aber sensitiv gegenüber Oxaliplatin zu sein.

In dieser AGEO-Studie wurde daher retrospektiv die Wirksamkeit der Kombinaiton von Fluoropyrimidin plus Oxaliplatin bei Patienten mit Kolonkarzinomen der Stadien II und III untersucht. Die 433 konsekutiven Patienten aus elf französischen Zentren (Alter: median 73
Jahre) hatten Tumoren mit einem Defekt in der Mismatch-Reparatur und wurden in kurativer Absicht operiert. 16,7\% der Patienten im Stadium II und 69,0\% derer im Stadium III erhielten eine adjuvante Chemotherapie aus einem Fluoropyrimidin mit oder ohne Oxaliplatin. Nach median 47 Monaten betrugen die Rezidivraten $5,7 \%$ bei den Stadium-IIund 20,9\% bei den Stadium-III-Patienten. Das krankheitsfreie 3-Jahres-Überleben (DFS) betrug in der Gesamtpopulation $76,2 \%$, bei alleiniger Operation $75,2 \%$, bei adjuvanter Chemotherapie mit einem Fluoropyrimidin $66,4 \%$ und bei Addition von Oxaliplatin 84,2\%. Damit verbesserte adjuvantes Oxaliplatin das DFS im Vergleich zur alleinigen Operation signifikant (Hazard Ratio [HR] 0,35; $<<0,001)$. Die alleinige Fluoropyrimidin-Therapie wirkte sich dagegen nicht signifikant auf das DFS aus
(HR 0,73; $\mathrm{p}=0$ 0,38). Das 3-Jahres-Gesamtüberleben von $85,3 \%$ wurde durch Addition von Oxaliplatin ebenfalls signifikant verlängert ( $H R 0,17, \mathrm{p}<0,001)$.

In den Subgruppenanalysen zeigte sich, dass der Nutzen der Oxaliplatinhaltigen Chemotherapie hinsichtlich des DFS bei Patienten mit Kolonkarzinom im Stadium III signifikant war (HR 0,41; $\mathrm{p}=0,02$ ), nicht aber die Gabe eines Fluoropyrimidins allein. Bei Tumoren im Stadium II zeigte sich ein Trend zu einen längeren DFS unter der Oxaliplatin-haltigen Chemotherapie, dagegen war die Chemotherapie ohne Oxaliplatin nicht mit dem DFS assoziiert.

Fazit: Diese Ergebnisse sprechen für die adjuvante Chemotherapie mit einem Fluoropyrimidin plus Oxaliplatin bei Patienten mit einem Kolonkarzinom im Stadium III mit einem Defekt in der Mismatch-Reparatur. Judith Neumaier

Tougeron D et al. Efficacy of Adjuvant Chemotherapy in Colon Cancer With Microsatellite Instability: A Large Multicenter AGEO Study. J Natl Cancer Inst. 2016;108(7):djv438.

\section{Hochrisiko-Kolonkarzinom: Wem nützt die adjuvante Chemotherapie?}

\author{
Welche Subgruppen profitieren bei einem Hochrisiko-Kolonkarzinom im \\ Stadium II nach der chirurgischen Resektion des Tumors von einer adjuvanten \\ Chemotherapie? Dieser Frage gingen niederländische Forscher nach.
}

Bs ei Hochrisiko-Kolonkarzinom im Stadium II (pT4, schlecht oder undifferenziert, Gefäßinvasion, Operation unter Notfallbedingungen und/oder $<10$ untersuchte Lymphknoten) kommt postoperativ eine adjuvante Chemotherapie in Betracht. In zwei Studien führte dies zu einem verlängerten Gesamtüberleben (OS), allerdings beschränkt auf $\mathrm{Pa}$ tienten mit Tumoren im Stadium III. Im Stadium II empfehlen einige Leitlinien ebenfalls eine adjuvante Chemotherapie, obwohl ihr Nutzen hier nicht eindeutig belegt ist. Eine Beobachtungsstudie sollte Klarheit schaffen.

Aufgenommen wurden alle 4.940 Patienten mit Hochrisiko-Kolonkarzinom im Stadium II, die zwischen 2008 und 2012 im niederländischen Krebsregister aufge- führt und operiert worden waren (medianes Alter 73 Jahre, 16\% mit adjuvanter Chemotherapie).

Das OS war nur bei Patienten mit pT4Tumoren oder mindestens zwei Risikofaktoren unter adjuvanter Therapie länger als ohne (3-Jahres-OS bei pT4-Tumor: 91 vs. $73 \%$; Hazard Ratio [HR] 0,43]). Auch das relative Sterberisiko (RER „relative excess risk“) war bei pT4-Patienten unter Chemotherapie niedriger (Relatives 3-Jahres-Überleben: 94 vs. 85\%; RER 0,36), ebenso bei mindestens zwei Risikofaktoren (86 vs. $75 \%$; RER 0,52). Für Patienten mit Notoperation fiel wahrscheinlich aufgrund der geringen Patientenzahl nur ein Trend zu verbessertem Überleben nach adjuvanter Chemotherapie auf. Bei Patienten mit nur schlecht oder ganz un- differenzierten Tumoren oder weniger als 10 untersuchten Lymphknoten gab es keinen signifikanten Zusammenhang zwischen adjuvanter Chemotherapie und Überleben.

Die weitere Unterteilung der Patienten mit mindestens zwei Risikofaktoren zeigte, dass die adjuvante Chemotherapie nur bei Patienten mit pT4-Tumor das Gesamtüberleben verlängerte (HR 0,48), aber nicht bei Patienten mit Nicht-pT4Tumor.

Fazit: Beim Patienten mit einem Hochrisiko-Kolonkarzinom im Stadium II verlängert die adjuvante Chemotherapie nur bei pT4-Tumoren das Überleben. Um unnötige Toxizitäten zu vermeiden, wäre eine weitere Präzisierung von Subgruppen sinnvoll.

Judith Neumaier

Verhoeff SR et al. Adjuvant chemotherapy is not associated with improved survival for all highrisk factors in stage II colon cancer. Int J Cancer. 2016;139(1):187-93. 